\title{
GOLDIE CONDITIONS FOR ORE EXTENSIONS OVER SEMIPRIME RINGS
}

\author{
André Leroy ${ }^{\dagger}$ and Jerzy Matczuk ${ }^{\ddagger}$ \\ † Université d'Artois, Faculté Jean Perrin \\ Rue Jean Souvraz 62307 Lens, France \\ E-mail: leroy@euler.univ-artois.fr \\ †Institute of Mathematics, Warsaw University, \\ Banacha 2, 02-097 Warsaw, Poland \\ E-mail: jmatczuk@mimuw.edu.pl
}

\begin{abstract}
Let $R$ be a ring, $\sigma$ an injective endomorphism of $R$ and $\delta$ a $\sigma$ derivation of $R$. We prove that if $R$ is semiprime left Goldie then the same holds for the Ore extension $R[x ; \sigma, \delta]$ and both rings have the same left uniform dimension.
\end{abstract}

\section{Introduction}

Throughout the paper $R$ will always denote an associative ring with unity. $R[x ; \sigma, \delta]$ will stand for the Ore extension of $R$, where $\sigma$ is an injective endomorphism and $\delta$ a $\sigma$-derivation of $R$.

It is well-known that when $R$ is semiprime and $\sigma$ is an automorphism then the Ore extension $R[x ; \sigma ; \delta]$ is a semiprime left (right) Goldie ring if and only if the ring $R$ is such and then $R$ and $R[x ; \sigma, \delta]$ have the same uniform dimensions. On the other hand, the easiest examples of left but not right Ore domains can be constructed as Ore extensions of the form $R[x ; \sigma]$, where $R$ is a field and $\sigma$ is an injective endomorphism of $R$ which is not onto. In such a case left uniform dimensions of $R$ and $R[x ; \sigma]$ are equal to one but right uniform dimensions of those rings are equal to one and infinity, respectively. 
The aim of the paper is to show that when $R$ is a semiprime left Goldie ring and $\sigma$ is injective then the Ore extension $R[x ; \sigma, \delta]$ is also semiprime left Goldie and both rings have the same left uniform dimensions. Contrary to the automorphism case, the fact that $R[x ; \sigma, \delta]$ is semiprime left Goldie and $R$ is semiprime does not imply that $R$ is left Goldie.

In section 2 below, with the help of certain classification results concerning injective endomorphisms and skew derivations, obtained by Cauchon and Robson (Cf. [CR]), we study Jordan extensions. In particular, the Jordan extensions of semisimple rings are completely described. These extensions are important tools for proving our main results in section 3 . In this section we also show that the ring $R$ and its Jordan extension $A$ have the same left uniform dimension provided $R$ is semiprime left Goldie.

\section{Jordan Extensions}

Let $R$ be ring with a fixed injective endomorphism $\sigma$. We say that an over-ring $A$ of $R$ is a Jordan extension of $R$ if $\sigma$ extends to an automorphism of $A$ and $A=\bigcup_{i=0}^{\infty} \sigma^{-i}(R)$. In this case we will write $R \subseteq_{\sigma} A$.

Jordan showed (Cf. [J] $)$, with the use of left localization of the Ore extension $R[x ; \sigma]$ with respect to the set of powers of $x$, that for any pair $(R, \sigma)$, such an extension $A$ always exists. Then he studied the passage of various algebraic properties from $R$ to $A$.

It is easy to observe that the Jordan extension $A$ of $R$ is an universal object, i.e. if $R \subseteq_{\sigma} A$ and $R \subseteq_{\sigma} A^{\prime}$ are two Jordan extensions of $R$ then the rings $A$ and $A^{\prime}$ are isomorphic, by an isomorphism which is identity on $R$.

In this section we will analyse the Jordan extension and collect some basic facts preparing the ground for results contained in the next section.

Throughout the paper $\mathbb{N}$ will denote the set of all natural numbers and $\mathbb{N}_{0}$ will stand for the set of all nonnegative integers.

If $\sigma$ is an endomorphism of the ring $R$, then $\sigma$ naturally induces the endomorphism $M_{n}(\sigma)$ of the full $n \times n$ matrix ring $M_{n}(R)$.

For an invertible element $u \in R, I_{u}$ will denote the inner automorphism of $R$ adjoint to $u$, i.e. $I_{u}(r)=u^{-1} r u$ for all $r \in R$.

Lemma 2.1. Suppose that $R \subseteq_{\sigma} A$ is a Jordan extension and $u \in R$ is an invertible element. Then:

1. For any $n \in \mathbb{N}, M_{n}(R) \subseteq_{M_{n}(\sigma)} M_{n}(A)$ is a Jordan extension.

2. If $\tau$ is an automorphism of $A$ such that $\sigma \tau=\tau \sigma$, then $\tau(R) \subseteq_{\sigma} A$ is a Jordan extension. If additionally $\tau(R) \subseteq R$ then $R \subseteq_{\sigma \tau} A$ is also a Jordan extension. 
3. $R \subseteq_{I_{u} \sigma} A$ is a Jordan extension.

Proof. The statements (1) (2) are clear.

(3) Obviously $I_{u} \sigma$ is an automorphism of $A$ such that $I_{u} \sigma(R) \subseteq R$.

Let $a \in A$. Then there exists $n \in \mathbb{N}$ such that $\sigma^{n}(a) \in R$ and, consequently, $\left(I_{u} \sigma\right)^{n}(a)=u^{-1} \sigma\left(u^{-1}\right) \ldots \sigma^{n-1}\left(u^{-1}\right) \sigma^{n}(a) \sigma^{n-1}(u) \ldots \sigma(u) u \in R$ follows.

Proposition 2.2. Let $A$ be an over-ring of a ring $R$ and $\sigma$ an injective endomorphism of $R$. The following statements are equivalent:

1. For all $k \in \mathbb{N}_{0}$ and $n \in \mathbb{N}, \sigma^{k}(R) \subseteq \subseteq_{\sigma^{n}} A$ is a Jordan extension.

2. For all $n \in \mathbb{N}, R \subseteq_{\sigma^{n}} A$ is a Jordan extension.

3. $R \subseteq_{\sigma} A$ is a Jordan extension.

4. There exists $n_{0} \in \mathbb{N}$ such that $R \subseteq_{\sigma^{n_{0}}} A$ is a Jordan extension.

5. There exist $k_{0} \in \mathbb{N}_{0}$ and $n_{0} \in \mathbb{N}$ such that $\sigma^{k_{0}}(R) \subseteq_{\sigma^{n_{0}}} A$ is a Jordan extension.

Proof. We only need to prove the implication (5) $\Rightarrow(1)$. Suppose that $k_{0} \in \mathbb{N}_{0}$ and $n_{0} \in \mathbb{N}$ are such that $\sigma^{k_{0}}(R) \subseteq_{\sigma^{n_{0}}} A$ is a Jordan extension. In view of Lemma 2.1(2), it is enough to show that $R \subseteq_{\sigma} A$ is a Jordan extension. For doing so, let us extend the injective endomorphism $\sigma$ of $R$ to an automorphism $\bar{\sigma}$ of $A$. By assumption, we know that for any $a \in A$ there exists $l(a) \in \mathbb{N}$ such that $b:=\left(\sigma^{n_{0}}\right)^{l(a)}(a) \in \sigma^{k_{0}}(R)$. Then we set $\bar{\sigma}(a):=\left(\sigma^{n_{0}}\right)^{-l}(\sigma(b)) \in A$. This definition makes sense, i.e. it does not depend on the choice of $l(a)$, since $\sigma^{n_{0}}$ is an automorphism of $A$.

It is easy to check that $\bar{\sigma}$ is an endomorphism of $A$ such that for any $r \in R \quad \bar{\sigma}(r)=\sigma(r)$ and $\sigma^{-n_{0}}(\sigma(r))=\bar{\sigma}\left(\sigma^{-n_{0}}(r)\right)$. Using this, one can check that $(\bar{\sigma})^{n_{0}}=(\sigma)^{n_{0}}$. This yields that $\bar{\sigma}$ is an automorphism of $A$ such that $\sigma=\left.\bar{\sigma}\right|_{R}$. Then it is clear that $R \subseteq_{\sigma} A$ is a Jordan extension.

Proposition 2.3. Let $R$ be a ring with an injective endomorphism $\sigma$. Then:

1. If $R$ is a simple artinian ring and $R \subseteq_{\sigma} A$ is the corresponding Jordan extension, then $A$ is also simple artinian.

2. Suppose that there is a finite bound on the cardinality of sets of orthogonal idempotents of $R$. Thus $R=\prod_{i=1}^{n} R_{i}$ is a finite product of indecomposable rings. Then:

(a) There exists a permutation $\rho \in \mathcal{S}_{n}$ of the index set $\{1, \ldots, n\}$ such that $\sigma\left(R_{i}\right) \subseteq R_{\rho(i)}$. 
(b) There exists $l \in \mathbb{N}$ such that for any $1 \leq i \leq n$, the restriction $\sigma_{i}^{l}$ of $\sigma^{l}$ to $R_{i}$ is an injective endomorphism of $R_{i}$.

(c) Suppose that $l \in \mathbb{N}$ is as in (b) above and for any $1 \leq i \leq n$, $R_{i} \subseteq_{\sigma_{i}^{l}} A_{i}$ is a Jordan extension. Then $\sigma$ can be extended to an automorphism of $\prod_{i=1}^{n} A_{i}$ and $\prod_{i=1}^{n} R_{i} \subseteq \sigma \prod_{i=1}^{n} A_{i}$ is a Jordan extension.

Proof. (1) Suppose that $R$ is simple artinian and $R \subseteq_{\sigma} A$ is the corresponding Jordan extension. If $R=D$ is a division ring, then it is known and easy to show that $A$ is also a division ring in this case. Let $R=M_{n}(D)$ where $D$ is a division ring. Then, by Theorem 2.4 of $\mathrm{CR}$, there exists an endomorphism $\tau$ of $D$ and an invertible element $u \in R$ such that $\sigma=I_{u} M_{n}(\tau)$. Let $K$ be the division ring such that $D \subseteq_{\tau} K$ is a Jordan extension. Then, by Lemma 2.1(1) and (3), we easily obtain that $M_{n}(D) \subseteq \subseteq_{\sigma} M_{n}(K)$ is a Jordan extension. This means that $A=M_{n}(K)$ is simple artinian.

(2) Suppose that $R=\prod_{i=1}^{n} R_{i}$.

The statement (a) is exactly Lemma 1.1 from [CR].

(b) Let $l$ denote the order of $\rho$ in $\mathcal{S}_{n}$. By (a) above $\sigma^{l}\left(R_{i}\right) \subseteq R_{i}$ for any $1 \leq i \leq n$ and clearly the restriction $\sigma_{i}^{l}$ is monic. Now, by Theorem 1.3 CR applied to $\sigma^{l}$, we obtain $\sigma_{i}^{l}\left(e_{i}\right)=e_{i}$, where $e_{i}$ denotes the unity of $R_{i}$.

(c) Let $1 \leq i \leq n$ and $l$ be as in (b). In view of (b), we can consider the Jordan extensions $R_{i} \subseteq \sigma_{i}^{l} A_{i}$ for $1 \leq i \leq n$. Then $\prod_{i=1}^{n} R_{i} \subseteq_{\sigma^{l}} \prod_{i=1}^{n} A_{i}$ is also a Jordan extension. Now, Proposition 2.2 completes the proof.

The above Proposition gives us immediately the following:

Corollary 2.4. Let a Jordan extension $R \subseteq_{\sigma} A$ of a semisimple ring $R$ be given. Then $A$ is semisimple. In fact, if $R=\prod_{i=1}^{n} M_{n_{i}}\left(D_{i}\right)$ for some division rings $D_{i}$, then $A=\prod_{i=1}^{n} M_{n_{i}}\left(K_{i}\right)$ for suitable division rings $K_{i}$.

For a semiprime left Goldie ring $R, Q(R)$ will denote the classical left quotient ring of $R$. Recall that, by Goldie's Theorem, $Q(R)$ is a semisimple ring.

The following results will be useful for our purposes.

Proposition 2.5. Let $R$ be a semiprime left Goldie ring with an injective endomorphism $\sigma$ and $R \subseteq_{\sigma} A$ be the corresponding Jordan extension. Then:

1. $\sigma(\mathcal{C}) \subseteq \mathcal{C}$, where $\mathcal{C}$ denotes the set of all regular elements of $R$.

2. $\sigma$ can be uniquely extended to an injective endomorphism of $Q(R)$.

3. $A$ is a semiprime left Goldie ring and $Q(R) \subseteq_{\sigma} Q(A)$ is a Jordan extension. 
4. Every $\sigma$-derivation $\delta$ of $R$ has a unique extension to a $\sigma$-derivation of $Q(R)$.

Proof. The first statement is a special case of a result of Jategaonkar (Cf. Proposition 2.4 [Ja] which states that $\sigma(\mathcal{C}) \subseteq \mathcal{C}$ when $R$ has left artinian quotient ring. (2), (4). The fact that $\sigma$ can be extended to $Q(R)$ is a wellknown consequence of (1). Then, it is also known that every $\sigma$-derivation $\delta$ of $R$ extends uniquely to a $\sigma$-derivation of $Q(R)$ and $\delta\left(c^{-1}\right)=-\sigma\left(c^{-1}\right) \delta(c) c^{-1}$ for all $c \in \mathcal{C}$.

(3). We know that $Q(R)$ is semisimple. Let $Q(R) \subseteq_{\sigma} A(Q(R))$ be a Jordan extension for $\sigma$ extended to $Q(R)$. Then, by Corollary 2.4 $A(Q(R))$ is also semisimple.

For any $x \in A(Q(R))$ there exist $n \in \mathbb{N}, c \in \mathcal{C}$ and $r \in R$ such that $\sigma^{n}(x)=c^{-1} r$, i.e. $x=\sigma^{-n}\left(c^{-1}\right) \sigma^{-n}(r)$. This shows that $A:=\bigcup_{i=0}^{\infty} \sigma^{-i}(R) \subseteq$ $A(Q(R))$ is a left order in the semisimple ring $A(Q(R))$. Now, Theorem 3.1.7 in [MR] yields that $A$ is semiprime left Goldie. Then $A(Q(R))=Q(A)$ easily follows.

The statement (3) from the above proposition was also obtained, using other methods, by Jordan in [Jo] (see [Jo Corollary 7.5, Proposition 7.1 and Theorem 7.2). The above proof was given both for completeness of the presentation and as an application of Corollary 2.4.

The following proposition shows that in case the $\sigma$-derivation $\delta$ is $q$-quantized, i.e. $\delta \sigma=q \sigma \delta$ for some central, $\sigma$ and $\delta$ invariant element $q \in R$, a Jordan extension $R \subseteq_{\sigma} A$ leads to a Jordan extension $R[x ; \sigma, \delta] \subseteq_{\sigma} A[x ; \sigma, \delta]$.

Proposition 2.6. Let $R \subseteq_{\sigma} A$ be a Jordan extension and $\delta$ be a q-quantized $\sigma$-derivation of $R$. Then:

1. $\delta$ can be uniquely extended to a q-quantized $\sigma$ derivation of $A$.

2. $\sigma$ can be extended to an injective endomorphism of $R[x ; \sigma, \delta]$. Moreover, $R[x ; \sigma, \delta] \subseteq_{\sigma} A[x ; \sigma, \delta]$ is a Jordan extension.

Proof. (1). Suppose that $\bar{\delta}$ is an extension of $\delta$ to a $q$-quantized $\sigma$-derivation of $A$. Let $a \in A$ and $n \in \mathbb{N}_{0}$ be such such that $\sigma^{n}(a) \in R$. Then $\bar{\delta}(a)=$ $q^{-n} \sigma^{-n}\left(\bar{\delta}\left(\sigma^{n}(a)\right)\right)=q^{-n} \sigma^{-n}\left(\delta\left(\sigma^{n}(a)\right)\right)$. This shows that $\bar{\delta}$ is uniquely determined by $\delta$ and $\sigma$.

Notice also that if $a \in A$ and $n, m \in \mathbb{N}_{0}$ are such that $\sigma^{n}(a), \sigma^{m}(a) \in R$, then $q^{-n} \sigma^{-n}\left(\delta\left(\sigma^{n}(a)\right)\right)=q^{-m} \sigma^{-m}\left(\delta\left(\sigma^{m}(a)\right)\right)$. Now, it is standard to check that $\delta: A \rightarrow A$ given by $\delta(a)=q^{-n} \sigma^{-n}\left(\delta\left(\sigma^{n}(a)\right)\right)$, where $n \in \mathbb{N}_{0}$ is such that $\sigma^{n}(a) \in R$ is a well defined $q$-quantized $\sigma$-derivation of $A$.

(2). The fact that $\sigma$ can be extended to an injective endomorphism of $R[x ; \sigma, \delta]$ is part of folklore: just define $\sigma(x)=q^{-1} x$. 
Let $p=\sum_{i=0}^{l} a_{i} x^{i} \in A[x ; \sigma, \delta]$. By assumption, there are $n_{i} \in \mathbb{N}_{0}$, with $0 \leq$ $i \leq l$, such that $\sigma^{n_{i}}\left(a_{i}\right) \in R$. Then $\sigma^{n}(p) \in R[x ; \sigma, \delta]$ where $n=\max \left\{n_{i} \mid 0 \leq\right.$ $i \leq l\}$. This easily yields that $R[x ; \sigma, \delta] \subseteq \subseteq_{\sigma} A[x ; \sigma, \delta]$ is a Jordan extension.

\section{Main Results}

We begin this section with a description of skew polynomial rings over semisimple rings.

Lemma 3.1. Let $R$ be a semisimple ring, $\sigma$ and $\delta$ an injective endomorphism and a $\sigma$-derivation of $R$, respectively. Then either

1. $R$ is simple artinian and there exists a division ring $D$ with an endomorphism $\sigma^{\prime}$ and $\sigma$-derivation $\delta^{\prime}$ such that $R[x, \sigma, \delta] \simeq M_{m}\left(D\left[y, \sigma^{\prime}, \delta^{\prime}\right]\right)$ for some $m \in \mathbb{N}$.

or

2 There exists a ring decomposition $R[x ; \sigma, \delta]=\prod_{j=0}^{k} B_{j}\left[x_{j}, \sigma_{j}, \delta_{j}\right]$ such that:

(a) $\sigma_{j}$ is an injective endomorphism of $B_{j}$ for any $1 \leq j \leq k$.

(b) If for some $1 \leq j \leq k \quad B_{j}$ is not a simple ring, then $\delta_{j}=0$.

Proof. (1) Suppose $R$ is simple artinian. Then $R=M_{m}(D)$ for some division ring $D$ and $m \in \mathbb{N}$ and the statement (1) is a particular case of Theorem 3.2 from $\mathrm{CR}$.

(2) Let $1=e_{1}+\ldots+e_{n}$ be the decomposition of 1 into the sum of central primitive orthogonal idempotents of $R$. Then $R=\prod_{i=1}^{n} R e_{i}$ and each $R e_{i}$ is simple artinian. By Proposition 2.3(2)(a), $\sigma$ induces a permutation $\rho$ of the index set $\{1, \ldots, n\}$. Let $\mathcal{O}_{1}, \ldots, \mathcal{O}_{k}$ denote the orbits of this action and set $B_{j}=\prod_{i \in \mathcal{O}_{j}} R e_{i}$ for $1 \leq j \leq k$. Let $\sigma_{j}, \delta_{j}$ be the restriction of $\sigma$ and $\delta$ to $B_{j}$. Then, by Theorem 1.3 from [CR], $\sigma_{j}$ is an injective endomorphism of $B_{j}$ and $\delta_{j}$ is a $\sigma_{j}$-derivation of $B_{j}$. Therefore, we can decompose the ring $R[x ; \sigma, \delta]$ in the following way:

$$
R[x ; \sigma, \delta]=\prod_{j=1}^{k}\left(B_{j}\left[y_{j} ; \sigma_{j}, \delta_{j}\right]\right) .
$$

When the cardinality $\# \mathcal{O}_{j}$ of the orbit $\mathcal{O}_{j}$ is equal to 1 , then $B_{j}$ is simple and we can set $x_{j}=y_{j}$ in this case. When $\# \mathcal{O}_{j}>1$ then, by Lemma $1.4\left[\mathrm{CR}\right.$, $\delta_{j}$ is an inner $\sigma_{j}$-derivation of $B_{j}$, i.e. there is $b \in B_{j}$ such that $\delta(r)=b r-\sigma(r) b$ for any $r \in B_{j}$. Then $B_{j}\left[y_{j} ; \sigma_{j}, \delta_{j}\right]$ is isomorphic to the ring $B_{j}\left[x_{j} ; \sigma_{j}\right]$, where $x_{j}=y_{j}-b$. This completes the proof of the lemma. 
In the sequel, the left uniform dimension of a $\operatorname{ring} R$ is denoted by $\operatorname{udim} R$. The following result was obtained by Mushrub in $[\mathrm{Mu}]$. The original argument was lengthy, thus we present a new very short proof.

Lemma 3.2. Let $R$ be a ring and $R \subseteq_{\sigma} A$ a Jordan extension associated to an injective endomorphism of $R$. Then $\operatorname{udim} R[x ; \sigma]=\operatorname{udim} A[x ; \sigma]=\operatorname{udim} A$.

Proof. Let $S=\left\{x^{n} \mid n \geq 0\right\}$. It is known and easy to check (Cf. [Jo]) that $S$ is a left Ore set in $R[x ; \sigma]$ and $S^{-1} R[x ; \sigma]$ is isomorphic to $A\left[x, x^{-1} ; \sigma\right]=$ $S^{-1} A[x ; \sigma]$. The left uniform dimension is preserved under left localizations with respect to Ore sets of regular elements (Cf. Lemma 2.2.12 [MR]). Hence $\operatorname{udim} R[x ; \sigma]=\operatorname{udim} A[x ; \sigma]$ follows.

Now, as $\sigma$ is an automorphism of $A$, a classical result of Shock (Cf. [Sh], Ma ) says that $\operatorname{udim} A=\operatorname{udim} A[x ; \sigma]$.

Lemma 3.3. Let $R$ be a semisimple ring, $\sigma$ an injective endomorphism and $\delta$ a $\sigma$-derivation of $R$. Then $\operatorname{udim} R[x ; \sigma, \delta]=\operatorname{udim} R$.

Proof. Recall that if a ring $B$ is isomorphic to $\prod_{j=1}^{k} B_{j}$, then $\operatorname{udim} B=$ $\sum_{j=1}^{k} \mathrm{udim} B_{j}$. Hence, in virtue of Lemma 3.1, it is enough to prove the lemma in two special cases: when $\delta=0$ and when $R$ is simple artinian.

Case 1. Suppose that $\delta=0$, i.e. $R[x ; \sigma, \delta]=R[x ; \sigma]$. Let $R \subseteq \subseteq_{\sigma} A$ be the Jordan extension. Since $R$ is semisimple, Corollary 2.4 implies that $\operatorname{udim} A=\operatorname{udim} R$. The thesis is now clear, thanks to Lemma 3.2

Case 2. Suppose that $R$ is simple artinian, i.e $R \simeq M_{m}(D)$ for some division ring $D$. Thus, by Lemma 3.1 the ring $R[x ; \sigma, \delta]$ can be presented in the form $M_{m}\left(D\left[y ; \sigma^{\prime}, \delta^{\prime}\right]\right)$. Since $D$ is a division ring, $D\left[y ; \sigma^{\prime}, \delta^{\prime}\right]$ is a principal left ideal domain, so $\operatorname{udim} D\left[y ; \sigma^{\prime}, \delta^{\prime}\right]=1$ and $\operatorname{udim} R[x ; \sigma, \delta]=m=\operatorname{udim} R$ follows. This completes the proof.

Theorem 3.4. Let $R$ be a semiprime left Goldie ring with an injective endomorphism $\sigma$ and $R \subseteq \sigma$ A be the corresponding Jordan extension. Then for any $\sigma$-derivation $\delta$ of $R \operatorname{udim} R[x ; \sigma, \delta]=\operatorname{udim} R=\operatorname{udim} A$.

Proof. By Proposition 2.5, $\sigma$ extends to an injective endomorphism of $Q(R)$ and $\delta$ extends to a $\sigma$-derivation of $Q(R)$. Thus, we can consider the ring extension $R[x ; \sigma, \delta] \subseteq Q(R)[x ; \sigma, \delta]$.

Clearly all elements from the set $\mathcal{C}$ of all regular elements of $R$ are invertible in $Q(R)[x ; \sigma, \delta]$ and every element from $Q(R)[x ; \sigma, \delta]$ can be presented in the form $c^{-1} p$ for some $c \in \mathcal{C}$ and $p \in R[x ; \sigma, \delta]$. This means that $\mathcal{C}$ is a left Ore set in $R[x ; \sigma, \delta]$ and $\mathcal{C}^{-1}(R[x ; \sigma, \delta])=Q(R)[x ; \sigma, \delta]$. Now, by [MR Lemma 2.2.12, we obtain:

$$
\operatorname{udim} R[x ; \sigma, \delta]=\operatorname{udim} Q(R)[x ; \sigma, \delta] \text { and } \operatorname{udim} R=\operatorname{udim} Q(R)
$$


By Proposition 2.5 we know that $A$ is semiprime left Goldie, so we also have $\operatorname{udim} A=\operatorname{udim} Q(A)$.

Notice that the same proposition yields that $Q(R) \subseteq_{\sigma} Q(A)$ is a Jordan extension. Since both $Q(R)$ and $Q(A)$ are semisimple rings, Corollary 2.4 and Lemma 3.3 give us

$$
\operatorname{udim} Q(R)=\operatorname{udim} Q(A) \text { and } \operatorname{udim} Q(R)[x ; \sigma, \delta]=\operatorname{udim} Q(R)
$$

respectively. This implies the thesis.

Mushrub in $[\mathrm{Mu}]$ investigated the left uniform dimension of skew polynomial rings $R[x ; \sigma]$, where $\sigma$ is an injective endomorphism of $R$. He proved, in particular, that when $R$ is a left Ore domain (i.e. a domain with $\operatorname{udim} R=1$ ), then $R[x ; \sigma]$ is a left Ore domain. He also constructed a series of examples showing that:

1. For any $n \in \mathbb{N}$ there is a commutative ring $R$ (not semiprime) with an injective endomorphism $\sigma$, such that $\operatorname{udim} R=n$ and $\operatorname{udim} R[x ; \sigma]=1$.

2. There exists a domain $R$ with an injective endomorphism $\sigma$ such that $\operatorname{udim} R[x ; \sigma]=1$ but $R$ has infinite both left and right uniform dimensions.

He posed a question whether $\operatorname{udim} R=\operatorname{udim} R[x ; \sigma]$ provided $R$ is a semiprime ring of finite left Goldie dimension. The above theorem gives a positive answer to this question for $R$ satisfying the ACC on left annihilators. We also have the following:

Corollary 3.5. Let $R$ be a left Ore domain with an injective endomorphism $\sigma$. Then $R[x ; \sigma, \delta]$ is a left Ore domain, for any $\sigma$-derivation $\delta$ of $R$.

Proof. Since $\sigma$ is injective, $R[x ; \sigma, \delta]$ is a domain. Now the thesis is a direct consequence of Theorem 3.4

Using Lemma 3.1 one can easily show that when $R$ is a semiprime left Goldie ring then the skew polynomial ring $R[x ; \sigma, \delta]$ is semiprime provided $\sigma$ in injective. The following lemma is slightly more general.

Proposition 3.6. Suppose that the ring $R$ satisfies the ACC on left annihilators. Let $\sigma$ and $\delta$ stand for an injective endomorphism and a $\sigma$-derivation of $R$, respectively. Then the Ore extension $R[x ; \sigma, \delta]$ is prime (semiprime), provided $R$ is prime (semiprime). 
Proof. Let $I$ be an ideal of $R[x ; \sigma, \delta]$. For any $n \geq 0$ define $I_{n}=\{a \in R \mid$ either $a=0$ or $a$ is the leading coefficient of some polynomial from $I$ of degree $n\}$. Clearly $\left\{I_{n}\right\}_{n \geq 0}$ is an ascending sequence of left ideals of $R$ such that $\sigma^{l}\left(I_{n}\right) \subseteq I_{n+l}$ for any $n, l \geq 0$. Since $R$ satisfies the ACC on left annihilators, it satisfies the DCC on right annihilators. Therefore, there exists $n_{0} \geq 0$ such that $\operatorname{rann}_{R}\left(I_{n}\right)=\operatorname{rann}_{R}\left(I_{2 n}\right)$ for any $n \geq n_{0}$.

Suppose that $R$ is prime and let $J$ be a nonzero ideal of $R[x ; \sigma, \delta]$. By the considerations above, there is $m \geq 0$ such that for any $n \geq m \operatorname{rann}_{R}\left(I_{n}\right)=$ $\operatorname{rann}_{R}\left(I_{2 n}\right)$ and $J_{n} \neq 0$. Assume $I J=0$. Then $I_{n} \sigma^{n}\left(J_{n}\right)=0$. This means that $\sigma^{n}\left(J_{n}\right) \subseteq \operatorname{rann}_{R}\left(I_{n}\right)=\operatorname{rann}_{R}\left(I_{2 n}\right)$ and $I_{2 n} \sigma^{n}\left(J_{n}\right)=0$ follows. Since $\sigma^{n}\left(I_{n}\right) \subseteq I_{2 n}$, we get $\sigma^{n}\left(I_{n} J_{n}\right)=\sigma^{n}\left(I_{n}\right) \sigma^{n}\left(J_{n}\right)=0$. This leads to $I_{n} J_{n}=0$ for any $n \geq m$, as $\sigma$ is injective. Since $J_{n} \neq 0$, primeness of $R$ yields $I_{n}=0$ for all $n \geq m$ and $I=0$ follows. This shows that $R[x ; \sigma, \delta]$ is prime.

The same argument applied to $J=I$ shows that $R[x ; \sigma, \delta]$ is semiprime provided $R$ is semiprime.

In the case $\sigma$ is an automorphism then the Ore extension $R[x ; \sigma, \delta]$ is always prime when $R$ is prime. However semiprimeness of $R$ does not imply semiprimeness of $R[x ; \sigma, \delta]$. In the case $\sigma$ is an injective endomorphism, the situation is more complex, as the following example shows. Namely, there exists a prime ring $R$ such that $R[x ; \sigma, \delta]$ is not semiprime.

Example 3.7. Let $R$ be a subset of $\mathbb{N} \times \mathbb{N}$ matrices over a field $K$ defined as follows $R=\left\{M \mid M=\sum_{i, j=1}^{n} a_{i j} e_{i j}+a \sum_{i=n+1}^{\infty} e_{i i}\right.$ for some $n \in \mathbb{N}$ and $\left.a_{i j}, a \in K\right\}$, where $\left\{e_{i j}\right\}_{i, j \in \mathbb{N}}$ denotes the set of matrix units. Then $R$ is a prime unital ring and it is easy to check that the map $\sigma: R \rightarrow R$ given by

$$
\sigma\left(\sum_{i, j=1}^{n} a_{i j} e_{i j}+a \sum_{i=n+1}^{\infty} e_{i i}\right)=a e_{11}+\sum_{i, j=1}^{n} a_{i j} e_{i+1, j+1}+a \sum_{i=n+2}^{\infty} e_{i i}
$$

is an injective endomorphism of $R$.

Notice that $e_{11} \sigma(R)=K e_{11}$. Therefore, for any $k \geq 0$, we have in $R[x ; \sigma]$ :

$$
e_{11} x R x^{k} e_{11}=K e_{11} e_{2+k, 2+k} x^{k+1}=0 .
$$

This shows that $e_{11} x R[x ; \sigma]$ is a nilpotent left ideal of $R[x ; \sigma]$, i.e. $R[x ; \sigma]$ is not semiprime.

Let $R \subseteq_{\sigma} A$ be the Jordan extension where $R$ and $\sigma$ are as in the above example. Then, by Proposition [2.6, we can consider the Jordan extension $R[x ; \sigma] \subseteq_{\sigma} A[x, \sigma]$. Then, as we have seen, $R[x ; \sigma]$ is not semiprime but $A[x, \sigma]$ is a prime ring since $A$ is prime and $\sigma$ is an automorphism of $A$.

We close the paper with the following: 
Theorem 3.8. Let $R$ be a semiprime left Goldie ring, $\sigma, \delta$ an injective endomorphism and a $\sigma$-derivation of $R$, respectively. Then $R[x ; \sigma, \delta]$ is also a semiprime left Goldie ring.

Proof. By Proposition 3.6 and Theorem 3.4 we know that $R[x ; \sigma, \delta]$ is a semiprime ring of finite left Goldie dimension. Thus, in order to complete the proof, it is enough to show that $R[x ; \sigma, \delta]$ satisfies the ACC on left annihilators. As we have seen in Proposition 2.5, $\sigma$ and $\delta$ can be extended to an injective endomorphism and a $\sigma$-derivation of $Q(R)$. Since the ACC on left annihilators is inherited on subrings, it is enough to prove that $Q(R)[x ; \sigma, \delta]$ satisfies the ACC on left annihilators. This means that without loss of generality we may assume that $R$ is semisimple. Then, by making use of Lemma 3.1, it is enough to consider only two cases: when $R$ is simple artinian and when $\delta=0$.

Case 1. Suppose $R$ is simple artinian. Then, by Lemma 3.1, $R[x ; \sigma, \delta]$ is isomorphic to $M_{m}\left(D\left[y, \sigma^{\prime}, \delta^{\prime}\right]\right)$ for some $m$, where $D$ is a division ring. $D\left[y, \sigma^{\prime}, \delta^{\prime}\right]$ is a left principal domain so, in particular, it is a prime left Goldie ring. Therefore, by Corollary 3.1.5 from [MR], $R[x ; \sigma, \delta]$ is a prime left Goldie ring, thus satisfies the ACC on left annihilators.

Case 2. Suppose that $\delta=0$, i.e. $R[x ; \sigma, \delta]=R[x ; \sigma]$. Let $R \subseteq_{\sigma} A$ be the Jordan extension. By Corollary [2.4, $A$ is also semisimple. Now $\sigma$ is an automorphism of $A$ so, by Theorem 2.6 of [Ma, $A[x ; \sigma]$ is a semiprime Goldie ring and $R[x ; \sigma]$ satisfies the ACC on left annihilators as a subring of $A[x ; \sigma]$.

If $\sigma$ is an automorphism of a semiprime ring $R$, then it is known (Cf. Theorem $2.6 \mathrm{Ma}$ ) that $R$ is semiprime left Goldie if and only if the Ore extension $R[x ; \sigma, \delta]$ is semiprime left Goldie. The quoted earlier example of Mushrub shows that when $\sigma$ is just an injective endomorphism, then the above equivalence does not hold. However, using Theorem 3.8, one can easily show that $R[x ; \sigma, \delta]$ is semiprime left Goldie if and only if $R$ is semiprime left Goldie provided $R$ is semiprime and $\operatorname{udim} R$ is finite.

\section{References}

[CR] G. Cauchon, J.C.Robson, Endomorphisms, derivations and polynomial rings, J. Algebra 53, (1978), 227-238.

[Ja] A.V. Jategaonkar, Skew polynomial rings over orders in Artinian rings, J. Algebra 21, (1972), 51-59.

[Jo] D.A. Jordan, Bijective extensions of injective rings endomorphisms, J. London Math. Soc. 25, (1982), 435-448. 
[Ma] J. Matczuk, Goldie rank of Ore extensions, Comm. Algebra 23(4), (1995), 1455-1471.

[MR] J.C. McConnel, J.C. Robson, Noncommutative noetherian rings, John Willey \& Sons Ltd, (1987).

[Mu] Mushrub V. A., On the Goldie dimension of Ore extensions with several variables (in Russian, English, Russian summary) Fundam. Prikl. Mat. 7(4), (2001), 1107-1121.

[Sh] R.C. Shock, Polynomial rings over finite dimensional rings, Pacific J. Math. 42, (1972), 251-257. 